\title{
Valérie AMIRAUX
}

Professeure agrégée, département de sociologie, Université de Montréal

Titulaire de la Chaire de recherche du Canada en études

du pluralisme religieux et ethnicité, rattachée au CEETUM

\section{(2008)}

\section{"De l'Empire à la République: à propos de I'«islam de France»."}

Un document produit en version numérique par Jean-Marie Tremblay, bénévole, professeur de sociologie retraité du Cégep de Chicoutimi

Courriel: jean-marie_tremblay@uqac.ca

Site web pédagogique : http://www.uqac.ca/jmt-sociologue/

Dans le cadre de: "Les classiques des sciences sociales" Une bibliothèque numérique fondée et dirigée par Jean-Marie Tremblay, professeur de sociologie au Cégep de Chicoutimi

Site web: http://classiques.uqac.ca/

Une collection développée en collaboration avec la Bibliothèque

Paul-Émile-Boulet de I'Université du Québec à Chicoutimi

Site web: http://bibliotheque.uqac.ca/ 


\section{Politique d'utilisation de la bibliothèque des Classiques}

Toute reproduction et rediffusion de nos fichiers est interdite, même avec la mention de leur provenance, sans l'autorisation formelle, écrite, du fondateur des Classiques des sciences sociales, Jean-Marie Tremblay, sociologue.

Les fichiers des Classiques des sciences sociales ne peuvent sans autorisation formelle:

- être hébergés (en fichier ou page web, en totalité ou en partie) sur un serveur autre que celui des Classiques.

- servir de base de travail à un autre fichier modifié ensuite par tout autre moyen (couleur, police, mise en page, extraits, support, etc...),

Les fichiers (.html, .doc, .pdf, .rtf, .jpg, .gif) disponibles sur le site Les Classiques des sciences sociales sont la propriété des Classiques des sciences sociales, un organisme à but non lucratif composé exclusivement de bénévoles.

Ils sont disponibles pour une utilisation intellectuelle et personnelle et, en aucun cas, commerciale. Toute utilisation à des fins commerciales des fichiers sur ce site est strictement interdite et toute rediffusion est également strictement interdite.

L'accès à notre travail est libre et gratuit à tous les utilisateurs. C'est notre mission.

Jean-Marie Tremblay, sociologue

Fondateur et Président-directeur général, LES CLASSIQUES DES SCIENCES SOCIALES. 
Cette édition électronique a été réalisée par Jean-Marie Tremblay, bénévole, professeur de sociologie au Cégep de Chicoutimi à partir de :

\section{Valérie AMIRAUX}

"De l'Empire à la République: à propos de l'«islam de France»."

Un article publié dans la revue Cahiers de recherche sociologique, no 46, septembre 2008, pp. 45-60. Montréal : Département de sociologie, UQAM. Liber, Éditeur. Un numéro intitulé : "L'islam, l'Empire et la République."

[Autorisation formelle accordée le 7 septembre 2010 par Monsieur Rachad Antonius, directeur du numéro, de diffuser tous les articles de ce numéro de la revue dans Les Classiques des sciences sociales.]

Courriels: antonius.rachad@uqam.ca valerie.amiraux@umontreal.ca

Polices de caractères utilisée : Comic Sans, 12 points.

Édition électronique réalisée avec le traitement de textes Microsoft Word 2008 pour Macintosh.

Mise en page sur papier format : LETTRE US, $8.5^{\prime \prime} \times 11^{\prime \prime}$.

Édition numérique réalisée le 12 décembre 2011 à Chicoutimi, Ville de Saguenay, Québec. 


\section{Valérie AMIRAUX}

Professeure agrégée, département de sociologie, Université de Montréal Titulaire de la Chaire de recherche du Canada en études du pluralisme religieux et ethnicité, rattachée au CEETUM

\section{"De l'Empire à la République: à propos de l'«islam de France»".}

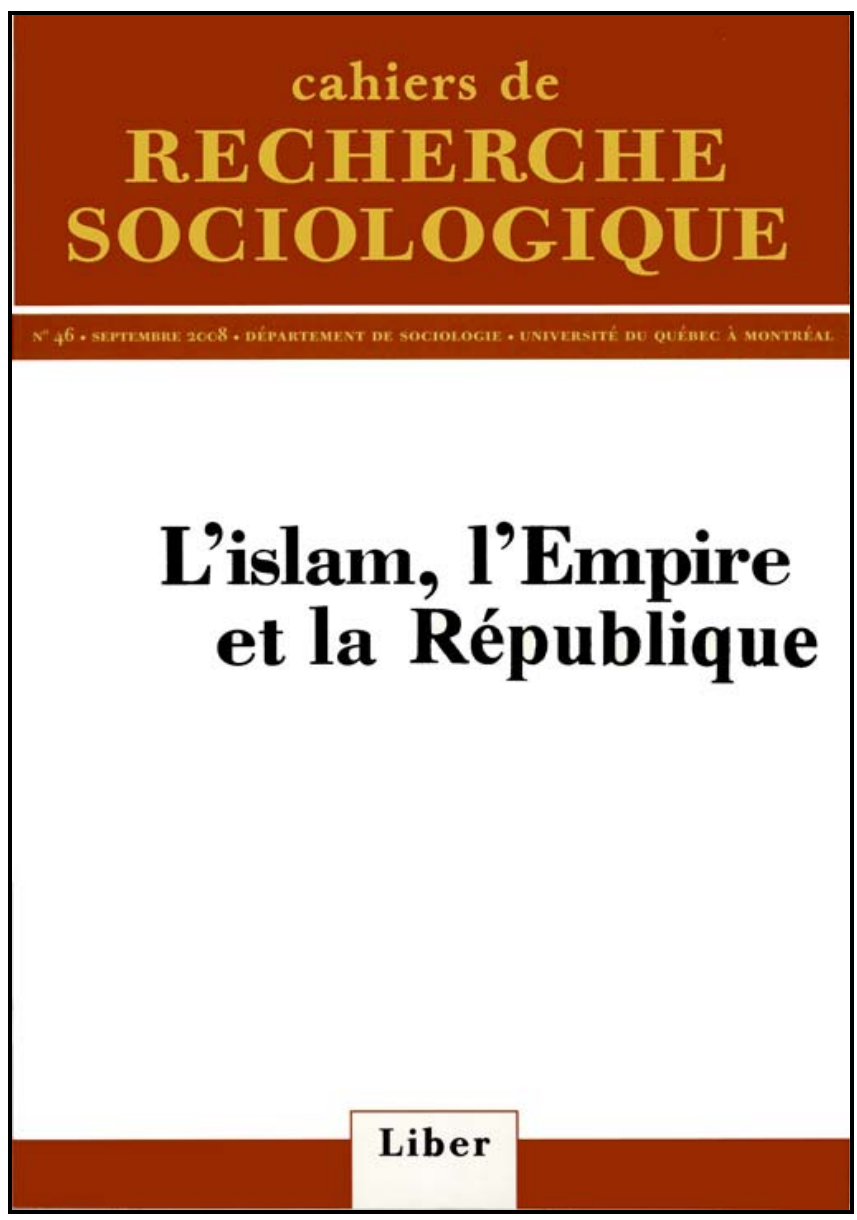

Un article publié dans la revue Cahiers de recherche sociologique, no 46, septembre 2008, pp. 45-60. Montréal : Département de sociologie, UQAM. Liber, Éditeur. Un numéro intitulé : "L'islam, l'Empire et la République." 


\section{Table des matières}

\section{Introduction}

L'Empire et la République: une histoire, plusieurs récits

Politiques musulmanes et laiccité

De l'indigène et du musulman, antithèses du citoyen?

Résumé / Abstract / Resumen 


\section{Valérie AMIRAUX}

Professeure agrégée, département de sociologie, Université de Montréal

Titulaire de la Chaire de recherche du Canada en études

du pluralisme religieux et ethnicité, rattachée au CEETUM

\section{"De l'Empire à la République: \\ à propos de l'«islam de France»".}

Un article publié dans la revue Cahiers de recherche sociologique, no 46, septembre 2008, pp. 45-60. Montréal : Département de sociologie, UQAM. Liber, Éditeur. Un numéro intitulé : "L'islam, l'Empire et la République."

\section{Introduction}

«Il y a des discussions qu'il ne faut pas prolonger indéfiniment. »

É. Durkheim

\section{Retour à la table des matières}

Après des années de comparaison entre les États membres de I'Union européenne quant au traitement de leurs minorités musulmanes (entre quinze et dix-sept millions de personnes ${ }^{1}$ ), l'évidence d'une forme de convergence se fait devant ce qui ressemble à un inéluctable affrontement des sociétés autour de différents sujets touchant à l'islam (culture et culte) et aux musulmans (croyants et citoyens). Le

1 Les chiffres cités dans ce texte sont extraits de J. Laurence et J. Vaisse, Integrating Islam. Political and Religious Challenges in Contemporary France, Washington, Brookings Institution Press, 2006. 
sujet de discorde le plus répandu concerne le port du foulard islamique dans les écoles publiques, voire dans l'espace public ${ }^{2}$. Quelques éléments permettent de nuancer les situations. Ce qui paraît alors distinguer la France laïque (cinq millions de musulmans dont trois sont français) d'autres contextes européens tient dans l'amplification croissante du caractère passionnel et quasi viscéral des débats, dans l'intensification des émotions (haine, mépris, ressentiment, amour, admiration) comme des enthousiasmes militants de la plupart des acteurs qui y prennent part depuis maintenant presque vingt ans ${ }^{3}$.

\section{[46]}

L'itinéraire de la laïcité est une longue trajectoire conflictuelle qui s'établit par paliers ou « seuils 4 ». Il n'y a donc rien d'incongru à voir le sujet continuer d'être débattu. Deux aspects nous semblent toutefois marquer la spécificité du moment actuel et nous ramènent à l'islam de France. Tout d'abord, l'implication de l'État et des autorités publiques dans la régulation des affaires pourtant intimes et privées de certaines populations, migrantes ou croyantes, en particulier musulmanes, se fait toujours plus active ${ }^{5}$. Parallèlement, la conjonction d'événements internationaux (surtout depuis le 11 septembre 2001) et

2 V. Amiraux, « Headscarves in Europe: What Is Really the Issue », dans S. Amghar, A. Boubekeur et M. Emerson, European Islam: The Challenges for Society and Public Policy, Bruxelles, ceps, 2007, p. 124-143.

3 La première affaire du foulard intervient à Creil, en région parisienne, en 1989. À propos de ces controverses ultramédiatisées, précisons que la plupart des acteurs ayant pris part aux discussions (des féministes, des femmes voilées, des jeunes musulmans, des intellectuels républicains, etc.), à l'exception notable des enseignants et des élèves, n'ont souvent jamais été dans une telle situation au sens ethnographique du terme, hors de la scène médiatique. Sur cette dernière, voir $P$. Tévanian, Le voile médiatique. Un faux débat : l'affaire du foulard islamique, Paris, Raisons d'agir, 2006.

4 J. Baubérot, Vers un nouveau pacte laïque? Paris, Seuil, 1990 ; et Laïcité $1905-$ 2005. Entre passion et raison, Paris, Seuil, 2004.

5 On pense ici au durcissement des lois sur le regroupement familial, aux campagnes de lutte contre les « mariages arrangés», confondus avec les « unions forcées», aux effets non anticipés des nouvelles politiques migratoires en Europe. Voir par exemple B. De Hart, « The Marriage of Convenience in European Immigration Law », European Journal of Migration and Law, vol. 8, nos 3-4, 2006, p. 251-262. 
l'impératif d'égalité de traitement de tous les cultes par la République ont accéléré le processus d'installation d'une instance de représentation nationale (adossée à des représentations régionales) du culte musulman qui, depuis avril 2003, existe sous la forme du Conseil français du culte musulman (CFCM $)^{6}$. Cette institutionnalisation de la représentation du culte musulman, tout en libérant un espace de parole au sein des populations musulmanes et plus largement dans la sphère publique, n'a pas pour autant neutralisé les dynamiques de stigmatisation, de racialisation de certains attributs de la diversité culturelle et cultuelle en lien avec l'islam ${ }^{7}$. Comment s'articule cette politique d'institutionnalisation à vocation égalitaire avec les expériences de discrimination et d'exclusion vécues par des musulmans vivant en France?

L'idée principale soutenue dans ce texte est que les assignations catégorielles sexuées et ethnicisées, opérées à partir d'une distinction religieuse, sont devenues autant de sources d'inégalité de traitement dans l'espace public et de qualification comme « déviance » aux normes républicaines du comportement citoyen. Si elles survivent dans le contexte républicain français, c'est parce qu'elles ont été historiquement naturalisées par le récit qui rattache l'Empire (colonial) à la République. Il s'agira donc ici de revenir [47] sur l'hypothèse de la continuité entre, d'une part, les imaginaires, discours et pratiques coloniaux et, d'autre part, les imaginaires, discours et pratiques contemporains de gestion de la diversité en France. Pour cela, nous nous arrêterons tour à tour sur le traitement de l'islam comme culte par les pouvoirs publics à partir d'un retour sur l'expérience coloniale, sur les modalités successives de régulation du culte musulman par les pouvoirs publics et sur l'hostilité de la République à certaines figures typifiant, ce qui pose problème, quotidiennement, dans l'interaction de la société avec l'altérité musulmane.

6 B. Godard et S. Taussig, Les musulmans en France. Courants, institutions, communautés : un état des lieux, Paris, Robert Laffont, 2006.

7 V. Amiraux et G. Jonker, «Introduction: Talking about Visibility - Actors, Politics, Forms of Engagement », dans V. Amiraux et $G$. Jonker (dir.), Politics of Visibilities. Young Muslims European Public Spaces, Bielefeld, Transcript Verlag, 2006, p. 9-20 ; D. Fassin et E. Fassin (dir.), De la question sociale à la question raciale? Représenter la société française, Paris, La Découverte, 2006. 


\section{L'Empire et la République: une histoire, plusieurs récits}

Retour à la table des matières

Dans l'épreuve publique contemporaine dont les musulmans vivant en France font aujourd'hui l'expérience 8 , les questions de représentation et de présentation de soi qui sont à l'oeuvre invitent à établir des liens directs avec l'histoire coloniale, relativement périphérique dans I'historiographie nationale ${ }^{9}$. La persistance de représentations colo-

8 Par « épreuve publique », j'entends ici l'entrecroisement de plusieurs dynamiques jouant à différentes échelles. Tout d'abord, celle qui construit le lien, historiquement et parfois de façon fantasmatique, entre islam et République française en termes d'incompatibilité, notamment autour des valeurs d'égalité et de laicité. Ensuite, celle qui, au cours des vingt dernières années (depuis 1989), a progressivement ethnicisé la différence confessionnelle des musulmans vivant en France au point de contribuer à la «culturalisation» des facteurs d'insécurité, aussi bien en lien avec l'international qu'avec les questions de politique intérieure. À ce mouvement, on associera par exemple la notion d'islamophobie. Enfin, celle qui, corollaire des deux précédentes, expose globalement tout individu musulman à une logique du soupçon, exigeant des preuves de bonne conduite, des démonstrations de loyauté et de connaissance des règles, jusque dans les interactions les plus quotidiennes de la vie. Les premiers éléments de cette réflexion ont été exposés dans V. Amiraux, «Expertises, savoir et politique. La constitution de I'islam comme problème public en France et en Allemagne », dans B. Zimmermann (dir.), Les sciences sociales à l'épreuve de l'action, Paris, EHESS, 2004, p. 209245.

9 L'ancrage politique des discussions en sciences sociales se confirme. Plusieurs auteurs ont récemment thématisé ces continuités jusqu'à parler de xénophobie d'État, notamment autour de la création du ministère de l'Identité nationale et de l'Immigration. "Par "xénophobie", j'entends l'ensemble des discours et des actes tendant à désigner l'étranger comme un problème, un risque ou une menace pour la société d'accueil et à le tenir à l'écart de cette société, que l'étranger soit loin et susceptible de venir ou déjà dans cette société, ou encore installé depuis longtemps. À partir de cette définition préalable, on peut distinguer une xénophobie de gouvernement ayant une histoire, des caractéristiques et des formes d'expression spécifiques de la xénophobie contestataire d'extrême droi- 
niales sur des populations dont [48] les États d'origine ont depuis accédé à l'indépendance, et les échos de ces représentations dans le débat sur l'intégration et la citoyenneté sont au coeur du discours politique et des travaux de sciences sociales depuis la fin des années 1990 10. Pour certains, le postcolonial serait partout mais toujours énoncé soit sur le mode de la dénonciation politique (comme par le mouvement des « indigènes » de la République), soit sur celui de la moquerie ${ }^{11}$. Selon d'autres, l'attention pour l'histoire coloniale et son imbrication avec le débat actuel sur le racisme et les discriminations s'appuient sur l'hypothèse que « les formes contemporaines de la question sociale seraient raciales parce qu'elles trouveraient leurs origines dans les pratiques et les schèmes de pensée de l'époque coloniale 12 ». Un ton dénonciateur domine les approches postcoloniales en France et exploite la trajectoire de l'indigène à l'immigré, insistant sur l'amnésie et la culpabilité face au passé colonial républicain. Quelques voix s'élèvent contre ces « indignations faciles 13 » et une " convocation de l'imaginaire colonial qui propose [...] une remémoration associant adroi-

te que l'Europe redécouvre depuis un peu plus de deux décennies » (J. Valluy, «Quelles sont les origines du ministère de I'Identité nationale et de I'Immigration? », Cultures et conflits, «Xénophobie de gouvernement, nationalisme d'État», vol. 69, 2008, p. 12). Sur cette question, voir aussi les numéros thématiques suivants: "Institutionnalisation de la xénophobie en France », AsyIon, vol. 4, 2008 ; «Identités nationales d'État», Journal des Anthropologues, 2007 : «Identité(s) nationale(s) : le retour des politiques de l'identité ?», Savoir/Agir, no 2, décembre 2007 ; «Choisir ses immigrés?», Raisons politiques, 26 mai 2007.

10 M. Breviglieri, «L'étreinte de l'origine. Attachement, mémoire et nostalgie chez les enfants d'immigrés maghrébins », Confluences Méditerranée, no 39, 2001, p. 37-47.

11 J. Stavo-Debauge, «L'invisibilité du tort et le tort de l'invisibilité », EspacesTemps.net, Actuel, avril 2007, http://www.espacestemps.net.

12 E. Saada, « Un racisme de l'expansion. Les discriminations raciales au regard des situations coloniales», dans D. Fassin et E. Fassin (dir.), op. cit., p. 56. En ce qui concerne les évolutions récentes des débats franco-français sur ces questions, on consultera Genèses, no 43, 2003, coordonné par I. Merle, et Genèses, no 69 , 2007, coordonné par E. Saada.

13 J. Stavo-Debauge, art. cité. 
tement le retour sur soi culpabilisant et l'autocritique disculpante ${ }^{14} \gg$. L'hostilité à l'instrumentalisation du passé se conçoit surtout devant la tentative d'en faire une ressource causale explicative englobante et sa tendance à la patrimonialisation du passé.

Il ne s'agit pas ici de réduire la complexité de l'expérience coloniale en matière de production de la différence et de catégorisation des individus à une lecture linéaire des formes de discrimination, de racisme et d'exclusion dont les musulmans seraient aujourd'hui les victimes, après l'avoir été sous l'administration coloniale. L'apposition artificielle d'une relation causale entre la manière d'administrer le culte musulman en 1830 en Algérie et [49] l'organisation d'une représentation de celuici en France en 2003 n'est pas le propos ${ }^{15}$. Les travaux de l'approche «postcoloniale», engagés récemment en France, focalisent rarement sur l'aspect religieux des choses. Pourtant, le cadrage de ces enjeux musulmans convoque l'idée du soupçon, de la nécessité du contrôle des hommes et de la maîtrise des lieux, du potentiel subversif de l'islam et des musulmans comme principale source d'insécurité et de désordre public ${ }^{16}$. Ils apparaissent comme des continuités tangibles, repérables dans le lexique administratif notamment ${ }^{17}$.

Aujourd'hui, S. Mazouz décrit finement à quel point le passage du statut d'étranger à celui de citoyen français ne se départit pas, lors des cérémonies de remise des décrets de naturalisation de la ville de Doucy, d'une forme de gêne liée à l'antinomie intrinsèque du cérémonial républicain. Ce rituel devient, par le truchement de l'embarras des représentants de l'autorité publique, une véritable épreuve de qualifica-

14 I. Merle et E. Sibeud, « Histoire en marge ou histoire en marche? La colonisation entre repentance et patrimonialisation », texte de l'intervention au colloque La politique du passé : constructions, usages et mobilisation de l'histoire dans la France des années 1970 à nos jours, 25-26 septembre 2003, http://histoiresociale.univ-paris1.fr/Collo/Merle.pdf.

15 Il n'y a là aucun moyen d'établir un lien univoque entre racisme colonial et discriminations contemporaines (E. Saada, art. cité, p. 64).

16 V. Geisser et A. Zemouri, Marianne et Allah. Les politiques françaises face à la "question musulmane», Paris, La Découverte, 2007, p. 15-41.

17 P. Le Pautremat, La politique musulmane de la France au XXe siècle. De I'Hexagone aux terres d'Islam, Paris, Maisonneuve et Larose, 2003 ; H. Laurens, Orientales II. La IIIe République et I'Islam, Paris, CNRS, 2004. 
tion 18. «Au moment même où il marque l'intégration, voire l'assimilation, des nouveaux naturalisés, il ne cesse de leur dire et de leur signifier qu'ils sont différents et illégitimes. [...] On peut parler d'antinomie parce que la manière dont sont construites ces cérémonies et la façon dont les représentants de l'État se réapproprient les catégories du droit marquent de plus belle la frontière qui sépare ce qui revient de droit et ce qui n'est jamais de droit ${ }^{19}$. »

\section{Politiques musulmanes et laïcité}

\section{Retour à la table des matières}

Si l'Empire est rêvé à l'image de la République 20 , l'expérience coloniale ne s'avère pas une mise en pratique fidèle des idéaux républicains. La conquête se fait bien au nom des principes républicains, et notamment du projet égalitaire universel, mais fait coexister un ensemble de pratiques dissonantes dont l'inégalitarisme entre citoyens de statut variable est une des plus [50] notoires, le moment phare de la mystique républicaine projetée dans l'acte colonial étant la fin du dix-neuvième siècle 21.

18 On sera alors peu surpris de savoir que les extensions abusives du domaine d'application de la loi de mars 2004 sur l'application du principe de laïcité dans les écoles publiques ont concerné l'interdiction aux salles municipales de femmes voilées venues recevoir ce décret des mains d'un élu ou d'un adjoint. Voir dans ce sens la délibération 2006-131 du 5 juin 2006 de la Haute Autorité de lutte contre les discriminations et pour l'égalité.

19 S. Mazouz, « Une célébration paradoxale. Les cérémonies de remise des décrets de naturalisation », Genèses, no 70, mars 2008, p. 89.

$20 \mathrm{~N}$. Bancel, P. Blanchard et F. Vergès, La République coloniale. Essai sur une utopie, Paris, Albin Michel, 2003.

21 Dans un langage plus contemporain, on a parlé, au sujet des failles du modèle d'intégration français, de la violence de l'universel abstrait qui exclut plus qu'il n'intègre, tout en aidant à la stigmatisation de l'autre ( $F$. Khosrokhavar, «L'universel abstrait. Le politique et la construction de l'islamisme comme une forme d'altérité », dans M. Wieviorka (dir.), Une société fragmentée: le multiculturalisme en débat, Paris, La Découverte, 1996, p. 113-151). 
Les pratiques coloniales en matière religieuse incarnent bien ces ambivalences. La loi de séparation de 1905 devient ainsi élément de la rhétorique de la mission civilisatrice (émancipatrice) dans les colonies et ressource de la domination coloniale. Détournée dans son esprit comme dans sa lettre, la loi de 1905 ne s'appliquera de fait jamais aux associations cultuelles musulmanes pourtant créées en vertu du décret de 1907. « Les oulémas pointèrent à cette occasion la contradiction de cette République prompte à faire de la laicité un dogme en métropole et à la dénaturer en Algérie dès lors que le contrôle de la population indigène était en jeu 22. "Tout en constituant l'un des fondements de la République et de sa culture politique, la laïcité est vidée de son contenu dans le contexte colonial, en particulier dans le cas algérien 23. Elle ne sera ainsi jamais appliquée en Algérie jusqu'en 1947 (même si elle y est transposée par décret depuis septembre 1907) et devient un ressort de contestation pour les réformistes musulmans. Il y a donc un énoncé, principalement greffé sur le modèle républicain de citoyenneté, et des pratiques distinctes selon que l'on se trouve en métropole ou dans les territoires colonisés. En métropole, la Troisième République met en place une démocratisation « intégratrice» des diverses composantes de la population française tandis que dans le monde extra-européen qu'elle domine la vision de la civilisation en marche se moule dans une exception coloniale ${ }^{24}$. Ces contradictions sont portées à leur comble dans le contexte algérien et rendues possibles par l'imprécision de la norme juridique concernant la citoyenneté. Jusqu'en 1946, les indigènes d'Algérie, s'ils sont bien de nationalité française, sont déniés de citoyenneté (ils ne votent pas) alors même que dans la

22 R. Achi, «L'islam authentique appartient à Dieu, I'islam algérien" à César. La mobilisation de l'association des oulémas d'Algérie pour la séparation du culte musulman et de l'État (1931-1956)», Genèses, no 69, 2007, p. 65.

23 R. Achi, «La séparation des Églises et de l'État à l'épreuve de la situation coloniale. L'État colonial et l'usage politique de la dérogation dans l'administration du culte musulman en Algérie (1907-1959) », Politix, vol. 17, no 66, 2004, p. 81-106.

24 Le terme «civilisation» a une histoire similaire dans les contextes coloniaux français et britanniques. Le mot civilization émerge autour de 1830 , et son usage au pluriel, plus tardif (1860), se construit en opposition avec l'idée de «barbares» et de "sauvages» (R. Williams, Keywords. Vocabulary of Culture and Society, Oxford, Oxford University Press, 1983). 
tradition juridique française race et ethnicité ne sont pas des catégories pour dire les droits civiques. En Algérie, pourtant département français, les Français musulmans [51] et les Français de métropole renvoient pour chaque groupe à deux vitesses d'un même régime de citoyenneté.

Il n'y a jamais eu de politique homogène vis-à-vis des musulmans des territoires conquis par l'entreprise coloniale. Les tensions entre le projet républicain et l'architecture complexe et inégalitaire du dispositif administratif colonial se complexifient au fil des conquêtes et de la confrontation avec des univers de sens très différents les uns des autres selon les territoires (Égypte, Maroc, Tunisie, Algérie). La politique musulmane de la France ne se met donc en place qu'en 1890. Produit du vingtième siècle, elle restera hantée par l'idée que tout ce qui se rattache à l'islam est potentiellement subversif et comporte des risques pour l'Empire. Les politiques de l'islam ont donc principalement souhaité, pour le dire vite, se muer en entreprises de contrôle visant à protéger le projet républicain et l'Empire de la menace «islam», et ce dans tous les domaines de la vie sociale. Après la politique arabe dont la conquête algérienne incarne l'idéal type avec la stratégie d'assimilation des nouveaux territoires au droit français tout en laissant à part le statut de l'indigène (français mais non citoyen) 25 - la «politique des égards », expression employée à la fin du dix-neuvième siècle, va désigner une pratique française dans la politique coloniale de direction des indigènes qui s'appuie sur certains d'entre eux et respecte des institutions traditionnelles 26 . La politique musulmane, sorte de

25 Le Code de l'indigénat est mis en place en Algérie en 1881. Il y est abrogé en 1946. Les indigènes musulmans, qui sont donc des Français de nationalité mais sans citoyenneté, deviennent alors des Français musulmans. L'ordonnance de mars 1944 portant sur le principe d'égalité des droits et des devoirs entre Français musulmans et non musulmans n'est effective qu'au prix d'une extrême lenteur, et, pendant la guerre d'Algérie, des clivages juridiques continueront de traverser la catégorie des citoyens français, le droit et ses usages renvoyant à des pratiques différentes ( $A$. Spire, «Semblables et pourtant différents. La citoyenneté paradoxale des Français musulmans d"'Algérie" en métropole », Genèses, no 53, décembre 2003, p. 61).

26 Dans l'acte de reddition de 1830, les autorités françaises s'engagent au respect des coutumes des populations locales, notamment le maintien d'un statut personnel fondé sur la loi religieuse et distinguant les différentes confessions présen- 
«police des âmes 27 », conjugue alors le savoir des universitaires, en particulier orientalistes, avec l'expérience de l'administration publique des territoires musulmans de l'Empire et, à partir des années 1910, celle des indigènes 28 . Les bureaux arabes, depuis 1833 en Algérie, et la Commission interministérielle des affaires musulmanes (CIAM), créée par décret en juin 1911, s'appuient ainsi sur des relais locaux pour assurer la médiation avec les autorités centrales. Initialement placée sous la tutelle du ministère des Affaires étrangères [52] (administrateur du Maroc, de la Tunisie et de la Syrie) et du ministère de I'Intérieur (responsable de l'Algérie), la CIAM a vocation à intégrer davantage la politique musulmane de la France. Elle est conçue comme une instance de coordination où la question de la représentation indigène est une constante depuis $1915^{29}$. Les postes de conseillers musulmans (« des intermédiaires susceptibles de pouvoir traduire les doléances et revendications des populations musulmanes $30 »)$ y seront créés en 1931 comme des vecteurs de la confiance vis-à-vis de la métropole: il faut bien montrer que la République n'est pas hostile à l'islam.

L'obsession de contrôle et d'organisation du culte musulman en conformité avec les principes républicains, qui voit véritablement le

tes sur le sol algérien. À compter du décret Crémieux en octobre 1870, les Israélites indigènes des départements d'Algérie deviennent des citoyens français, laissant les musulmans seuls à leur statut d'indigènes.

27 C. Liauzu, L'Europe et l'Afrique méditerranéenne, de Suez (1869) à nos jours, Bruxelles, Complexe, 1994, p. 61.

28 H. Laurens, op. cit., p. 251-280.

29 Sur ce point, nous renvoyons à P. Le Pautremat, op. cit.

30 « Il nous est impossible de prétendre à une influence immédiate sur des peuples pour qui nous sommes des étrangers. Nous avons besoin, pour les diriger, d'avoir des intermédiaires : nous devons leur donner des chefs, sans quoi ils s'en choisiront eux-mêmes. J'ai préféré les ulémas et les docteurs de la loi : 10 parce qu'ils l'étaient naturellement: 20 parce qu'ils sont les interprètes du Coran, et que les plus grands obstacles que nous avons éprouvés et que nous éprouverons encore proviennent des idées religieuses : 30 parce que ces ulémas ont des moeurs douces, aiment la justice, sont riches et animés d'un bon principe de morale. [...] Je les ai intéressés à mon administration. Je me suis servi d'eux pour parler au peuple » (extrait du Mémoire sur l'administration intérieure de l'Égypte de Bonaparte, cité par H. Laurens, op. cit., p. 54). 
jour au tournant des années 1980-1990 sous la forme d'un projet d'institutionnalisation de la représentation du culte, n'est donc pas une invention exclusive de la Cinquième République. Elle a une trajectoire bien antérieure, quoique justifiée par des fins différentes. Au lendemain des indépendances et dans le contexte des migrations de maind'oeuvre jusque vers le milieu des années 1970, c'est une phase d'un relatif laisser-faire qui prévaut tout d'abord, à l'intersection d'une vision pragmatiste des enjeux, de préoccupations d'ordre sécuritaire et de positionnements diplomatiques vis-à-vis du monde arabe. À partir de 1989-1990, phase dont la mise en place du CFCM en 2003 est l'aboutissement, alternent des périodes de tensions puis d'entente qui scandent le rythme de l'institutionnalisation de la représentation du culte musulman ${ }^{31}$. Pour nombre de musulmans engagés dans la consultation créée par Jean-Pierre Chevènement lorsqu'il était ministre de l'Intérieur (de 1997 à 2000), les discussions avec les pouvoirs publics portent les traces du colonialisme. La charge d'illégitimité qui pèse sur les besoins des musulmans en matière de culte, ou plutôt le soupçon de défaut de loyauté qui les suit, se manifeste lors de la définition du cadre normatif [53] dans lequel conduire les discussions. L'invitation faite aux représentants du culte musulman de «se joindre à la table de la République», suivant l'expression de Chevènement utilisée de manière quasi liturgique par ses successeurs, s'accompagne en janvier 2000 de la signature, difficile, d'une déclaration d'intention relative aux droits et obligations des fidèles du culte musulman en France, rebaptisée par la suite Principe et fondements juridiques régissant les rapports entre les pouvoirs publics et le culte musulman en France. Les conseillers en charge de l'islam, qui se succèdent aux côtés des ministres depuis 1989, alternent quant à eux d'idéologues (« civiliser les musulmans ») à pragmatiques (au service d'« un réalisme sociologique et démographique»), explique V. Geisser. Pris dans la consultation,

31 L'institutionnalisation de l'islam en avril 2003 a produit une instance de représentation, le $\mathrm{cfcm}$, organe né pour partie d'élections, pour partie de nomination. Pour une synthèse efficace, voir J. Laurence et J. Vaisse, op. cit., p. 135-162 ; M. Zeghal, « La constitution du Conseil Français du Culte Musulman : reconnaissance politique d'un Islam français?», Archives de sciences sociales des religions, « La République ne reconnaît aucun culte », vol. 129, 2005, http://assr.revues.org/document1113.html. 
certains représentants parmi les plus jeunes reçoivent l'invitation des ministres de l'Intérieur successifs comme une «injonction coloniale [...] paternaliste », tandis que les plus anciens oscillent entre sentiment d'incompétence et méfiance, tous exprimant des affects mêlés 32 . L'homo islamicus 33 qui émerge à la croisée de la régulation publique et des exigences des associations musulmanes dans cette dynamique d'institutionnalisation est une figure Janus, comme dans le contexte colonial. Le premier visage est le produit d'une saisie institutionnelle de la religion musulmane comme culte, coulé dans le bronze de la virtì civique et du loyalisme à la République. L'autre visage est celui de la résistance à la pression normative qui s'exerce sur les représentants de l'islam au moment de s'asseoir à la table de la République, celui de la contestation. Plus que les altercations et autres rixes entre différents représentants associatifs et de fédérations de musulmans, on retiendra la dynamique de tutelle par les pouvoirs publics et les autorités politiques qui s'accélère au cours de 2002-2003 34. L'histoire de la relation entre l'État français (colonial et postcolonial) et le culte musulman est in fine davantage celle d'une interférence que d'une indifférence neutre. Si les interférences de l'État peuvent être souhaitables dans l'intérêt de la liberté commune, elles ne le sont pourtant qu'à la condition d'intervenir sans arbitraire sur des populations vulnérables 35 .

32 V. Geisser et A. Zemouri, op. cit., p. 71-99.

33 L. Tezcan, « Kultur, Gouvernementalität der Religion und der Integrationsdiskurs », dans M. Wohlrab-Sahr et L. Tezcan, Konfliktfeld Islam in Europa, BadenBaden, Nomos Verlag (Soziale Welt), 2007, p. 51-74; voir également sur ce terme, V. Geisser, «L'islamophobie en France au regard du débat européen », dans R. Leveau et K. Mohsen-Finan (dir.), Musulmans de France et d'Europe, Paris, CNRS, 2005, p. 67-69.

34 Cet aspect de l'institutionnalisation a été abondamment traité. Voir par exemple F. Frégosi, «Les enjeux liés à la structuration de l'islam en France », dans R. Leveau et K. Mohsen-Finan, op. cit., p. 99-114.

35 P. Pettit, Républicanisme: une théorie de la liberté et du gouvernement, Paris, Gallimard, 2004. 


\section{De l'indigène et du musulman, antithèses du citoyen?}

\section{Retour à la table des matières}

La tension entre principes et idéaux, d'une part, pratiques et actes, de l'autre, se mesure aussi au quotidien, la difficulté de la coexistence entre univers de sens et définitions du bien pouvant alors prendre la forme du malaise, de l'hostilité, de la discrimination, voire du racisme explicite. À une autre échelle de ce que nous venons d'esquisser sur le plan institutionnel, le début du vingt et unième siècle a vu s'opérer le renouvellement d'une iconographie de la déviance à l'idéal républicain, non plus fantasmée mais incarnée dans des figures masculines et féminines typifiant les comportements considérés comme hostiles à la République. Le lien est réalisé entre une iconographie fantasmée de l'altérité et un malaise beaucoup plus ordinaire, résultat d'interactions quotidiennes entre personnes ignorantes ou familières de l'islam. Cette appréhension quotidienne ne nous éloigne pas de l'hypothèse centrale de cet article (l'hypothèse de la continuité entre imaginaires, discours et pratiques pré et postcoloniaux dans la gestion de l'altérité musulmane en France). Elle lui donne un contenu plus ethnographique qu'historiographique, en regardant la façon dont la «gouvernementalité » des corps (au sens où s'engage une lutte pour le contrôle des modes d'intégration et de reproduction sociale entre État et acteur sociaux, qui passe par une gouvernance des corps des jeunes musulmans et musulmanes) permet de repérer des formes de continuité dans la stigmatisation de certaines pratiques ${ }^{36}$.

36 Pour une lecture parallèle de la stigmatisation des pratiques corporelles en lien avec la religion (la prière, le port du foulard) et de l'intégration par le sport, voir P. A. Silverstein, Algeria in France. Transpolitics, Race and Nation, Bloomington, Indiana University Press, 2004, en particulier p. 121-150; N. Guénif, « La réduction à son corps de l'indigène de la République», dans N. Bancel, P. Blanchard et S. Lemaire, La fracture coloniale, Paris, La Découverte, 2005, p. 199-209. 
Deux profils types restituent la figure de l'autre dans le contexte colonial, d'une part la figure du «sauvage» et de l'autre celle de l'« indigène 37 ». Dans cette synthèse de fantasmes incarnés dans des figures archétypiques, le sauvage musulman (le barbare) s'installe comme le négatif de l'homme blanc, civilisé et catholique ${ }^{38}$. Plus précisément, parmi [55] les multiples faces de l'indigène, "celle du cavalier maghrébin, perpétuant une tradition magnifiant la valeur guerrière de I'"Arabe", mais qui fixe définitivement sa fonction, sa perception et les craintes qu'il inspire (particulièrement, l'islam) dans le champ étroit du politique $39 »$, s'inscrit dans l'univers du politique et du revendicatif, inaugurant ainsi la trajectoire d'un stéréotype qui fonctionne aujourd'hui encore selon des modalités proches. Cette compétence au combat des Arabes, mise sous contrôle par la République, l'idée de leur nature violente et de leurs pulsions non contrôlables sont des éléments durables d'une trajectoire historique de stigmatisation au cours de laquelle l'« indigène musulman » ne se révèle pas aussi «éducable » qu'on l'avait projeté. Précisons en passant que, dans ce contexte, l'islam est moins appréhendé comme théologie que comme culture, voire plutôt comme non-culture ou inculture. Le "garçon arabe» (issu de l'immigration maghrébine), ultra-médiatisé ces dernières années comme fauteur de troubles et source d'insécurité dans et hors de sa communauté d'appartenance, "est un revenant du passé colonial [...] l'un des avatars de l'indigène devenu immigré puis musulman $40 »$, figure

37 N. Bancel et $P$. Blanchard, « Civiliser: l'invention de l'indigène », dans P. Blanchard et S. Lemaire (dir.), Culture coloniale. La France conquise par son Empire 1871-1931, Paris, Autrement, 2003, p. 149-162.

38 Plus intéressant peut-être que les spécificités d'une histoire nationale, il existe dans l'Occident européen du Moyen Âge une image de l'islam qui s'est très peu modifiée depuis. Sur l'histoire longue de la construction de représentations réciproques, inspirées par l'affrontement idéologique, l'animosité, la haine ('islamophobie dirait-on aujourd'hui), mais aussi l'amour ou la fascination, voir $\mathrm{N}$. Daniel, Islam et Occident, Paris, Le Cerf, 1993 ; J. Goody, L'Islam en Europe: histoire, échanges, conflits, Paris, La Découverte, 2004 ; J. Dakhlia, Islamicités, Paris, PUF, 2005.

39 N. Bancel et P. Blanchard, art. cité, p. 150.

40 N. Guénif, «La Française voilée, la beurette, le garçon arabe et le musulman laïque. Les figures assignées du racisme vertueux», dans N. Guénif (dir.), La République mise à nu par son immigration, Paris, La Fabrique, 2006, p. 118. 
parfois désirée au temps des colonies et honnie aujourd'hui ${ }^{41}$. La cruauté des Arabes, pilier des archétypes de la culture coloniale, se trouve réactualisée autour de nouvelles scènes de confrontation, à propos de pratiques sexuelles barbares qui défraient la chronique, registre bien différent du répertoire transnational largement dominé par le paradigme sécuritaire de l'adéquation entre islam et violence terroriste 42 . La stigmatisation se joue ici autour de l'incapacité des hommes à contrôler leur nature incivile, de «leur [56] inaptitude à intérioriser, dans leur corps, les règles de bienséance et de civilité qui régiraient depuis toujours le commerce entre hommes et femmes, expression de l'exception française $43 »$.

Dans un registre très différent mais non sans lien avec ce qui précède, le coup de tête de Zinedine Zidane pendant le match de la coupe du monde de football de 2006, geste de transgression publique des normes et codes de la sportivité, constitue, pour certains, l'aveu d'une incapacité à contrôler des émotions d'ordre privé, en total désaccord avec la noblesse attendue des sportifs de haut niveau. Yasmin Jiwani souligne l'imagerie orientaliste, en particulier animalière, que les journalistes de la presse internationale mobilisent pour décrire et interpréter l'événement et conclure à l'échec de la performance du sportif

41 Le champ des études postcoloniales n'a pas ouvert encore dans le contexte français de véritable chantier sur les questions de genre. Dans son magnifique ouvrage, Massad esquisse une histoire du tourisme homosexuel pour exposer les interactions entre les questions de «culture», de pratiques sexuelles et de puissance coloniale. Il reprend à son compte la critique que Stoler adresse à Foucault : «Pourquoi avons-nous si facilement adopté son interprétation d'un ordre sexuel qui, au dix-neuvième siècle, exclut le fait colonial ou l'incorpore comme un trait parmi d'autres » (A. Stoler, cité dans J. A. Massad, Desiring Arabs, Chicago, Chicago University Press, 2007, p. 7).

42 On pense ici à la médiatisation des affaires de viols en réunion aussi appelées « tournantes ». Voir L. Muchielli, Le scandale des tournantes, Paris, La Découverte, 2005. Sur l'imagerie populaire et la stigmatisation du « garçon arabe » comme hétérosexuel violent, polygame, exciseur, «voleur, violeur et voileur», voir $N$. Guénif et É. Macé, Les féministes et le garçon arabe, La Tour-d'Aigues, L'Aube, 2004. Sur la construction médiatique de l'adéquation entre l'islam et la violence terroriste, voir T. Delthombe, L'islam imaginaire. Les musulmans de France à la télévision 1975-2003, Paris, La Découverte, 2005.

43 N. Guénif, « La réduction à son corps de l'indigène... », art. cité, p. 204. 
sur la scène de l'intégration ${ }^{44}$. On retrouve par ailleurs dans la couverture médiatique de l'événement la même construction d'une image racialisée de la figure de l'homme arabe (ici kabyle) hétérosexuel dont l'une des vocations consiste en la protection de l'honneur des femmes de la famille insultées par le joueur de l'équipe adverse. Nacira Guénif avait finement décrit, dans son analyse des positions d'acteurs dans la controverse sur le port du foulard en 2003-2004, les figures rassurantes de l'intégration réussie que sont d'une part la «beurette » et de l'autre le «musulman laique» par opposition aux pôles négatifs (l'intégriste barbu et la jeune fille voilée).

Figures du miraculé sauvé par le sport (Zidane) ou de la réussite exceptionnelle par l'école (Rachida Dati, nommée garde des Sceaux par Nicolas Sarkozy en mai 2007), ces individus se révèlent faillibles et sont décrits comme empêtrés dans des «retours d'origines» qui les rattrapent et les font devenir des transgresseurs plutôt que des conformistes dans le contexte de la morale laïque, quand bien même l'islam n'est pas une de leurs identités affichées. La tension est à son comble entre le formalisme concret de la citoyenneté incarnée dans des droits et principes fondamentaux et un citoyen idéel qui n'a jamais pris racine au point de constituer une abstraction, de s'incarner dans une absence qui, pour certains, est aveu d'incompétence.

Entre culte de la virginité et propension au viol, la dangerosité de l'indigène, son insoumission et sa résistance ont pris le dessus - indépendamment de son sexe, convient-il d'ajouter. De fait, l'égalité de sexe est en quelque sorte réalisée dans le racisme ordinaire, qui désormais ne stigmatise pas seulement la figure masculine (l'Arabe musulman) mais aussi son équivalent féminin (la jeune femme voilée). Pour les hommes et les femmes, le "déficit d'intégration" de ces Français postcoloniaux ne viendrait $\mathrm{ni}$ de leur inadéquation sociale ni de leur inadaptation au marché de l'emploi. Il se logerait dans leur corps, dans

44 Y. Jeewani, «Sport as Civilizing Mission: Zinedine Zidane and the Infamous Head-Butt», Topia. Canadian Journal of Cultural Studies, vol. 19, printemps 2008, p. 11-33. 
leur incapacité à le [57] soumettre aux règles de l'autocontrainte prescrites par le processus de civilisation français 45 . »

La stigmatisation dont le foulard islamique est l'objet depuis vingt ans emprunte des itinéraires proches, et, là encore, les corps sont à l'avant-scène. La cristallisation sur le port du foulard en France a suivi plusieurs dynamiques, de la politisation à la juridicisation des discussions en sa faveur ou en sa défaveur ${ }^{46}$. Le consensus qui domine en 2003-2004, lorsque la controverse sur le port du foulard dans les écoles publiques culmine, est traversé par la certitude que le foulard est une nuisance, un élément nocif pour la République et pour les jeunes filles qui le portent. Sa présence contrevient à plusieurs principes centraux pour l'équilibre du dispositif républicain. Il exporte dans l'espace public des signes privés révélant l'appartenance confessionnelle des unes. L'identité croyante prime l'identité citoyenne rompant ainsi l'égalité entre élèves en introduisant un élément visible de distinction entre tous. Il nuit ainsi à la mission civique de l'école et conduit vers une "école à la carte» où interviennent d'autres autorités que celle des enseignants. Enfin, cinquième aspect, le foulard islamique contrecarre la liberté religieuse en nuisant à la liberté de conscience d'autrui 47 . Pour les défenseurs de la République laïque forteresse, le foulard est donc la trace évidente d'une menace pour l'ordre public, et pour l'écologie symbolique dans laquelle les expériences sensibles des citoyens prennent forme et font sens. La séparation entre État et Églises demeure le motif principal de la loi de mars 2004, sur lequel se greffent des arguments concernant la protection des jeunes filles voilées et les valeurs républicaines dont l'école est le principal lieu de transmission.

45 N. Guénif, « La Française voilée... », art. cité, p. 120.

46 V. Amiraux, « Headscarves in Europe... », art. cité ; J. Bowen, Why the French Don't Like Headscarves: Islam, the State, and Public Space, Princeton, Princeton University Press, 2006 ; F. Lorcerie, La politisation du voile : l'affaire en France, en Europe et dans le monde arabe, Paris, Harmattan, 2005 ; C. de Galembert (dir.), « Le voile en procès », Droit et société, no 68, 2008.

47 c. Laborde, «Secular Philosophy and Muslim Headscarves in Schools», The Journal of Political Philosophy, vol. 13, no 3, 2005, p. 305-329 ; voir en particulier p. 327-328. 
Dans le cadre républicain laïque français, la sphère intime et privée ne fait, politiquement, aucune différence dans la façon dont l'État connaît ses administrés. C'est précisément là, dans l'intime et le privé, que la religion se confine dans l'ensemble des démocraties libérales sécularisées. On dira donc ici, de manière synthétique, que le sécularisme, dont la laïcité est pour partie une déclinaison, relève d'une ambition libérale de protéger d'une part les libertés individuelles, de l'autre les différentes conceptions du bien qui cohabitent dans une société plurielle. Le coeur du sécularisme en contexte libéral articule dès lors dans un dispositif commun la liberté religieuse, l'égalité de tous les citoyens et la neutralité de l'État. La laïcité en France est en fait tour à tour érigée en principe régulateur de l'administration des différences dans l'espace public et en cadre de reconnaissance, [58] voire d'accommodement d'une réalité sociale plurielle ${ }^{48}$. La version contemporaine de la laïcité va cependant au-delà du point de référence nodal de la séparation. Dans le contexte français de la laïcité, deux éléments ont leur importance: d'une part, le rôle des institutions dans la mise en oeuvre de l'égalité et de la neutralité et, d'autre part, la «doctrine de conscience» qui prescrit des codes et des normes de conduite, aussi bien à l'attention des organisations religieuses ( une laicisation interne) que des individus, desquels on attend une retenue religieuse dans l'espace public ${ }^{49}$. Ce sont donc ces ancrages infrapolitiques d'attitudes républicaines qui rendent possibles la justice et la tolérance, non seulement par la médiation de la loi, mais aussi par l'effet d'une inscription éthique profonde dans la culture politique des individus.

Les musulmans se trouvent placés, en France, à l'intersection de deux injonctions contraires: l'une requiert l'invisibilité individuelle fai-

48 «...] c'est-à-dire une laïcité qui, tout en respectant l'autonomie respective de l'État et des religions et en veillant à garantir les principes fondamentaux de libertés et de non-discrimination qu'elle implique, reconnaît les apports sociaux, éducatifs et civiques des religions et les intègre de ce fait dans la sphère publique » (J.-P. Willaime, « Séparation et coopération Églises-État en Allemagne», dans A. Dierkens et J.-P. Schreiber (dir.), Laïcité et sécularisation dans I'Union européenne, Bruxelles, Éditions de l'université de Bruxelles, 2006, p. 89-105).

49 C. Laborde, «The Culture(s) of the Republic. Nationalism and Multiculturalism in French Republican Thought », Political Theory, vol. 29, no 5, 2001, p. 716-735. 
sant l'« éloge de l'immigré invisible $50 »$, l'autre invite à la reconnaissance par l'égalité de traitement des différents cultes présents sur le territoire. L'attente d'invisibilité se conjugue avec le projet émancipateur de privatisation des identités culturelles au nom de l'égalité de tous dans l'espace public citoyen, libre d'attachements émotionnels à des particularités culturelles contingentes. Cette invisibilité reste équivoque. Des formes d'identifications significatives pour certaines populations, individuellement et collectivement, sont ainsi tenues dans l'absence, l'invisibilité, ce qui constitue, explique J. Stavo-Debauge à propos des Noirs en France, une «expérience de l'humiliation, du mépris et du déni de reconnaissance le plus paradigmatique. En effet, être invisible, c'est ne pas être compté parmi les membres pléniers et autorisés d'une communauté, ou même d'une situation. C'est également ne pas être pris en compte comme un participant de plein droit pouvant apporter une contribution susceptible d'être reconnue distinctement et saluée par les autres participants de la communauté ${ }^{51}$." L'invitation à la reconnaissance quant à elle procède de la convergence en Europe autour du multiculturalisme et de la lutte contre les discriminations comme modalité politique de gestion de la diversité. Ce second point n'est pas dépourvu d'ambiguité dans le contexte français où, parmi les attributs [59] disqualifiés par le modèle républicain d'intégration et de conquête, la religion figure en bonne place. Or, l'« indigène musulman tend à devenir un client musulman, objet de toutes les sollicitudes des pouvoirs publics, aboutissant à l'enfermer dans son identité religieuse au mépris de ses autres appartenances sociales $52 »$.

La place de l'islam en France n'est pas stabilisée. Son destin se joue entre plusieurs enjeux (historiques, politiques, juridiques, sociaux) qui convergent tous vers le constat d'un déficit de confiance du dispositif républicain laïque. Comme l'a exposé Habermas dans sa conversation avec Benoît XVI, l'État laïque (sécularisé, dit Habermas) reposerait sur des présupposés dont il ne serait pas capable d'assurer la garantie

50 G. Noiriel, Immigration, antisémitisme et racisme en France (XIXe-XXe siècle): discours publics, humiliations privées, Paris, Fayard, 2007.

51 J. Stavo-Debauge, op. cit.

52 V. Geisser et A. Zemouri, op. cit., p. 11. 
et la perpétuation. L'orientation vers le bien commun présuppose plus qu'une simple obéissance aux lois et implique un engagement plus coûteux en termes de vertus politiques, explique-t-il, et l'on ne peut uniquement agir par intérêt ou par contrainte pour que se déploient des valeurs comme celles de la solidarité, de la tolérance et de la reconnaissance. La constance historique de l'hostilité à certaines formes exprimées de diversité, jusque dans les recoins de la vie intime des individus, n'est pas sans faire écho, à propos du discours républicain et de ses réflexes d'hostilité exprimés publiquement, à un nationalisme inavoué. Le racisme antimusulman profondément moderne qui caractérise les opinions publiques européennes s'appuie, dans le contexte français, sur l'universalisme républicain, "nouvelle incarnation de l'impérialisme postcolonial, qui fait de l'islam un "autre" inassimilable et confond l'autodétermination du sujet autonome avec la subjectivité de I'homme blanc européen 53 ».

Historiquement, le républicanisme postrévolutionnaire s'est notamment mis en place en France autour de la reconnaissance de la nécessité d'une privatisation des éléments culturels, en particulier religieux, pour permettre un traitement égalitaire de tous les citoyens dans l'espace public. Liberté de conscience, incompétence réciproque du politique et du religieux, égalité des religions et des convictions devant l'État, constituent quelques-uns des acquis fondamentaux de ce projet républicain qui se fait aussi laïque. Théoriquement, la clef de voûte du dispositif (au sens de l'ensemble des règles et des institutions en charge d'encadrer son application) tient dans la neutralité des pratiques du pouvoir et d'exercice de l'autorité publique. La neutralité devient alors l'indicateur de réalité politique de la laïcité dans sa capacité de faire face aux revendications et requêtes toujours renouvelées ${ }^{54}$. En pratique, l'éloignement de ces idéaux [60] s'est progressivement construit comme une normalité, et les tensions autour des questions relatives à la politique à tenir dans le contexte multiculturel

53 C. Laborde, «The Culture(s) of the Republic », art. cité, p. 721.

54 D. Koussens, «Le port de signes religieux dans les écoles québécoises et françaises. Accommodements (dé)raisonnables ou interdiction (dé)raisonnée? », Globe. Revue internationale d'études québécoises, vol. 10, no 2, et vol. 11, no 1, 20072008, p. 115-131. 
français ont contribué à « routiniser » l'idée que la laïcité serait un élément de la culture politique nationale toujours plus menacé, sans jamais cesser d'être un horizon idéal vers lequel tendre. En d'autres termes, la laïcité, que l'on accuse certains de transgresser alors que l'on voudrait qu'ils s'y conforment, reste une idée fondatrice de l'esprit républicain, une conviction partagée quoique souffrant d'interprétations multiples. Depuis mars 2004, le vote de la loi encadrant, en application du principe de laïcité, le port de signes ou de tenues manifestant une appartenance religieuse dans les écoles, collèges et lycées publics l'a instituée, pour reprendre la définition de Durkheim, « fait moral » au sens d'« une règle de conduite sanctionnée», de nature obligatoire, indépendamment de la diversité des consciences qui composent une société 55 . Dans un secteur au moins de la vie publique, la transgression de la laïcité est dorénavant sanctionnée. Cela peut-il suffire à tenir le sens premier du projet laïque postrévolutionnaire de pacification et de réconciliation?

55 Durkheim précise que les faits moraux suivent des règles qui se distinguent par deux traits : la société intervient lorsqu'un acte dévie de la règle morale à laquelle il doit se conformer ; la réaction suit l'infraction « avec une véritable nécessité », car «la réalité d'une obligation n'est certaine que si elle se manifeste par quelque sanction». Et d'ajouter, «les seuls progrès possibles sont ceux que la société fait collectivement » (É. Durkheim, De la division du travail social, Paris, Alcan, 1902 (1893), p. 16, 20 et 19). [Livre disponible dans Les Classiques des sciences sociales. JMT.] 


\section{Résumé}

Valérie Amiraux

De l'Empire à la République :

quelques réflexions à propos de l'« islam de France »

\section{Retour à la table des matières}

Cet article propose, à partir d'une approche inscrite dans un contexte historique, de réfléchir à l'hypothèse de la continuité des politiques musulmanes (imaginaires, [138] discours et pratiques coloniaux) de la France. Il avance que les assignations catégorielles sexuées et ethnicisées, opérées à partir d'une distinction religieuse, sont devenues autant de sources d'inégalité de traitement dans l'espace public et de qualification comme « déviance » aux normes républicaines du comportement citoyen. Celles-ci survivent dans le contexte républicain français parce qu'elles ont été historiquement naturalisées par le récit qui rattache l'Empire (colonial) à la République. On regardera successivement le traitement de l'islam comme culte par les pouvoirs publics à partir d'un retour sur l'expérience coloniale, sur les modalités successives de régulation du culte musulman par les pouvoirs publics et sur l'hostilité de la République à certaines figures typifiant ce qui pose problème, plus quotidiennement, dans l'interaction de la société avec l'altérité musulmane.

mots clés : imaginaires, colonial, islam, politique musulmane, France. 


\section{Abstract}

Valérie Amiraux

De l'Empire à la République :

quelques réflexions à propos de l'« islam de France»

\section{Retour à la table des matières}

This paper examines the hypothesis of the continuity of the French policies towards Islam (colonial imaginaries, discourses and practices) from the standpoint [141] of the historicity of such policies. It suggests that the assignment of ethnicised and gendered categories based on religious differentiations have become sources of inequality of treatment in the French public space and explain why some types of individuals have been considered as 'deviant' from the republican norms of behaviour of citizens. Such categories have survived in the French republican context, because they have been naturalized by narratives that link the (colonial) Empire to the Republic. We will examine the treatment of Islam as a religious denomination by public authorities, by going back to the colonial experience and to the successive modalities of the regulation of the Islamic religion by French authorities. We will examine the hostility of the Republic towards certain emblematic figures that constitute a daily problem in the interaction of society with the Muslim other.

keywords : Imaginaries, colonial, islam, Muslim politics, France 


\section{Resumen}

Valérie Amiraux

De l'Empire à la République :

quelques réflexions à propos de l'« islam de France»

\section{Retour à la table des matières}

Este artículo propone, a partir de un enfoque histórico, de poder reflexionar a la hipótesis de la continuidad de las políticas musulmanas (imaginarios, discursos y [144] prácticas colonialistas) de Francia. También propone que las asignaciones de categorización sexuadas y étnicas, realizadas a partir de una distinción religiosa, se han convertido tanto en fuentes de tratamientos desiguales en el espacio público como en calificativos de "desviación" con respecto a las normas republicanas del comportamiento ciudadano. Éstas últimas sobreviven en un contexto republicano francés porque han sido históricamente naturalizadas por el relato que liga el Imperio (colonial) a la República. Se observará sucesivamente el tratamiento del islam como culto por parte de los poderes públicos a partir de una vuelta a la experiencia colonial, sobre las modalidades sucesivas de regulación del culto musulmán por parte de los poderes públicos y sobre la hostilidad de la República a ciertas figuras que caracterizan la problemática cotidiana en la interacción de la sociedad con la alteridad musulmana.

palabras clave: Imaginario, colonial, Islam, política musulmana, Francia

Fin du texte 